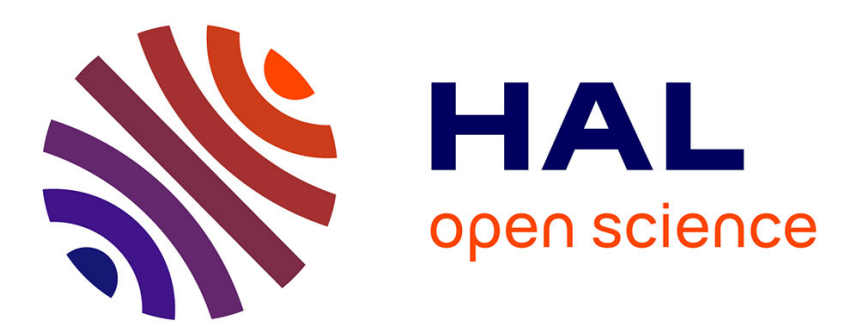

\title{
Semantic Evidential Lane Grids with Prior Maps for Autonomous Navigation
}

\author{
Chunlei Yu, Véronique Cherfaoui, Philippe Bonnifait
}

\section{To cite this version:}

Chunlei Yu, Véronique Cherfaoui, Philippe Bonnifait. Semantic Evidential Lane Grids with Prior Maps for Autonomous Navigation. 19th IEEE Intelligent Transportation Systems Conference (ITSC 2016), Nov 2016, Rio de Janeiro, Brazil. pp.1875-1881. hal-01378525

\section{HAL Id: hal-01378525 \\ https://hal.science/hal-01378525}

Submitted on 10 Oct 2016

HAL is a multi-disciplinary open access archive for the deposit and dissemination of scientific research documents, whether they are published or not. The documents may come from teaching and research institutions in France or abroad, or from public or private research centers.
L'archive ouverte pluridisciplinaire $\mathbf{H A L}$, est destinée au dépôt et à la diffusion de documents scientifiques de niveau recherche, publiés ou non, émanant des établissements d'enseignement et de recherche français ou étrangers, des laboratoires publics ou privés. 


\title{
Semantic Evidential Lane Grids with Prior Maps for Autonomous
}

\section{Navigation}

\author{
Chunlei $\mathrm{Yu}^{1}$, Veronique Cherfaoui $^{1}$, Philippe Bonnifait ${ }^{1}$
}

\begin{abstract}
Real-time modeling of the surrounding environment is a key functionality for autonomous navigation. Bird view grid-based approaches have interesting advantages compared to feature-based ones. Methods able to encode occupancy information and to manage perception uncertainty in dynamic environments are quite well known but very few studies have been carried out on encoding semantic information in grids. This kind of information can be crucial in many situations in order to make the vehicle able to follow basic road rules, such as lane keeping or lane changes. Usual approaches often detect lane markings using on-board cameras or lidars but the problem is tricky when the road is multi-lane or in challenging weather conditions. In this work, we propose to tackle this problem by using a vectorial prior map that stores detailed lane level information. From a given pose estimate provided by a localization system, we propose an Evidential model that encodes lane information into grids by propagating the pose uncertainty on every cell. This Evidential model is compared with a classical Bayesian one and some of its special characteristics are highlighted. Real results carried on public roads with the same real-time software are reported to support the comparison.
\end{abstract}

\section{INTRODUCTION}

Road scene understanding is a key task for autonomous navigation. Segmentation of the driving scene provides semantic view about the environment. In the literature, many scene segmentation algorithms rely on vision processing. Scene images are usually segmented into road, obstacle and sky. In [1], the authors propose a learning method for road scene segmentation from a single image; in [2], a holistic 3D scene understanding method uses geo-tagged images; in [3], the authors propose an algorithm to detect road segment combined with a prior geographical information.

For autonomous navigation, the only information of the road space is, however, not sufficient because vehicles need to know where it is authorized to go, and so, lane-level information is required. When there is an obstacle in the egolane, the host vehicle has two options: i) keep-lane and stop or ii) lane-change. The second alternative needs semantic road rule information to evaluate if the space is accessible or not. According to road rules, solid lane markings are normally forbidden to cross, whereas dashed ones indicate possible lane change. These lane marking types imply the road rules that should be obeyed by the host vehicle. Many lane detection methods have been studied and developed [4][5][6] for this purpose. Nevertheless, lane detection methods relying

\footnotetext{
1 The authors are with Sorbonne Universités, Université de Technologie de Compiègne, CNRS Heudiasyc UMR 7253, France
}

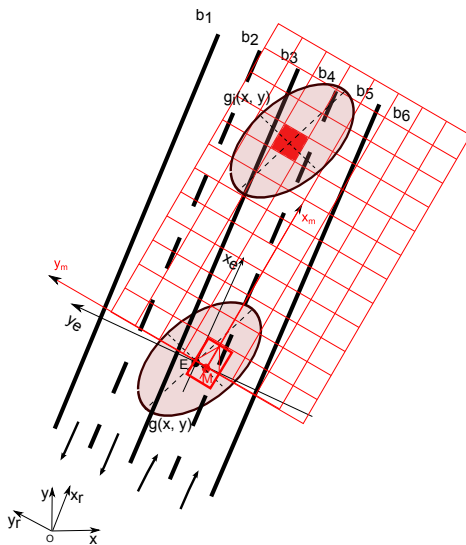

Figure 1: Illustrative Lane grid drawn in red. The goal is to characterize the lane membership to every cell according to the pose uncertainty.

only on vision and lidar systems perform badly in challenging weather conditions or parallel multi-lane conditions.

We propose to tackle this problem by using prior maps and accurate pose estimation. Indeed, more and more detailed and accurate geo-referenced databases become available. This kind of information source is very rich for autonomous navigation. Through the use of maps, one can have access to lane level attributes as long as the pose uncertainty is well handled. Moreover, a map feedback can also be used to refine the localization [7][8].

In this paper, a new method to represent semantic lane information and to encode it into spatial grids is presented. Inspired from occupancy grids [9][10][11] which focus on the management of obstacle information into grid cells, one can interpret lane information as semantic meaning encoded as well into the cells. Fig 1 shows an illustrative grid drawn in red. From the vehicle's point of view, the navigable space is made up of the accessible lanes having a driving direction compatible with the vehicle orientation. Lanes with opposite direction and space outside of the road are non-navigable. So, a minimal frame of discernment can be defined as $\Omega=\{$ Ego, Accessible, Forbidden $\}$. In lane grids, every cell is filled with a belief state which characterizes this semantic lane information. In order to retrieve the map information, the propagation of the pose uncertainty into the grid construction process has to be perfectly handled. This is presented in Section III. In order to encode semantic lane information, two approaches are developed in sections IV 
and $\mathrm{V}$. These Probabilistic and Evidential approaches are compared with real road data in section $\mathrm{VI}$

\section{SYSTEM WORK-FLOW AND FRAMES}

Fig 2 shows the proposed Lane Grid construction process. The Ego-localization system provides the pose information (position and heading) of the vehicle. The estimated pose has to be reliable which implies that it has to be consistent with the true pose (the covariance matrix of the estimation error has to be well estimated). There exists two kinds of uncertainty in the process which come respectively from the estimated pose and from the map. In the current approach, we consider that the map is accurate (the one used in the experiments has been made with high-grade mobile mapping sensors). The pose uncertainty is therefore the predominant uncertainty which has to be taken into account. The two steps for lane grid construction are illustrated in sections IV and $\mathrm{V}$

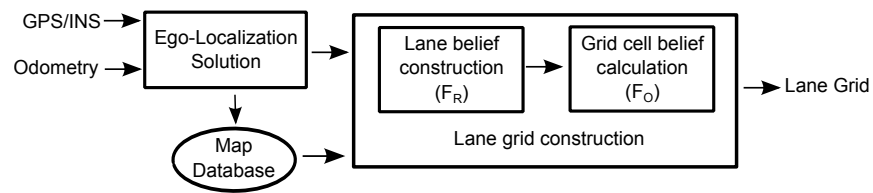

Figure 2: Lane Grid construction process

Let denote $F_{O}$ the global frame in which the coordinates of the geometry points of the map are defined. It has an origin $O$ close to the navigation area to work in 2D and $x$ and $y$ point towards East and North respectively. A road-oriented frame $F_{R}[8]$ has the same origin as the global frame but with its $\mathrm{x}$-axis pointing in the direction of the road. It is actually a rotated version of the global frame. The body frame $F_{M}$ of the vehicle is defined at the center of the vehicle's rear axle with an origin denoted $M$. Note that $M$ has not a deterministic location in $F_{O}$ or $F_{R}$ because of the estimation uncertainty. In Fig 3, a position $M$ with an uncertainty ellipse $g(x, y)$ is given for illustrating purpose.

\section{UnCERTAinty PROPAGation OVER THE GRID}

The term Belief used in Fig 2 can have different meanings depending on the considered approach. In the probabilistic approach, Belief refers to Probability; in the evidential one, Belief means Mass, since Mass is the basic belief assignment in the Dempster-Shafer's theory.

\section{A. Lateral position uncertainty in the road frame}

The lane states are characterized depending on which lane the host vehicle is located. This requires the knowledge of the lateral position with respect to the road. The pose is estimated in the global frame with a 2D ellipse uncertainty $g(x, y)$ as shown in Fig 3 . In the frame $\left(x_{e}, y_{e}\right)$ defined at the estimated position, the lateral position uncertainty to construct the lane belief distribution is perpendicular to the lane direction, i.e,

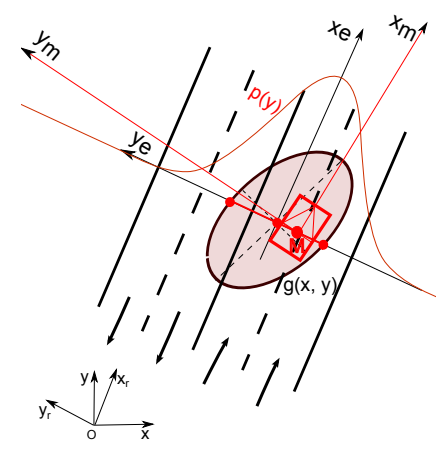

Figure 3: Lateral position uncertainty. The real pose is displayed by the red box.

along the axis $y_{e}$. In Fig 3, the red 1-D Gaussian $p(y)$ shows this uncertainty.

To calculate this uncertainty, suppose that the position uncertainty is represented in $F_{O}$ by the covariance matrix ${ }^{\circ} \mathbf{P}$ $(2 \times 2)$, the transformation of this uncertainty into the roadoriented frame $F_{R}$ is given by:

$$
{ }^{\mathbf{R}} \mathbf{P}=\left[\begin{array}{ll}
{ }^{R} p_{11} & { }^{R} p_{12} \\
{ }^{R} p_{12} & { }^{R} p_{22}
\end{array}\right]=\mathbf{R} \cdot{ }^{O} \mathbf{P} \cdot \mathbf{R}^{T}
$$

where $\mathbf{R}=\left[\begin{array}{cc}\cos (\psi) & \sin (\psi) \\ -\sin (\psi) & \cos (\psi)\end{array}\right]$ is the rotation matrix in which $\psi$ is the heading of the road. Then in $F_{R}$ the lateral Gaussian distribution's standard deviation can be computed by (see [8] for details):

$$
\sigma_{R}=\sqrt{{ }^{R} p_{22}} \cdot\left(1-\left(\frac{{ }^{R} p_{21}}{\sqrt{{ }^{R} p_{11}} \cdot \sqrt{{ }^{R} p_{22}}}\right)^{2}\right)
$$

\section{B. Propagation of pose uncertainty in the computation of lane cells belief}

Fig 1 illustrates the uncertainty in the computation of the belief distribution of the grid cells. $b_{1} \sim b_{6}$ represent the belief distribution of each lane (the case where the position distribution extends to outside of the road is taken into consideration, each side of the space outside the road is taken as a lane). To illustrate the model, let us imagine that the true position of vehicle is $M$ in Fig 1

Consider the uncertainty denoted $g_{i}(x, y)$ of the red cell $i$ displayed in Fig 1. The coordinates vector of cell $i$ in $F_{M}$ is

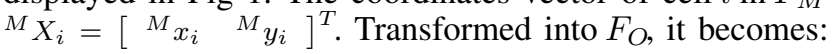

$$
{ }^{\mathrm{o}} \mathbf{X}_{\mathbf{i}}=\left[\begin{array}{c}
O_{x_{i}} \\
{ }^{O} y_{i}
\end{array}\right]={ }^{\mathrm{O}} \mathbf{R}_{\mathrm{M}} \cdot\left[\begin{array}{c}
{ }^{M} x_{i} \\
{ }^{M} y_{i}
\end{array}\right]+\left[\begin{array}{c}
O_{x_{M}} \\
{ }^{O} y_{M}
\end{array}\right]
$$

where ${ }^{\mathrm{O}} \mathbf{R}_{\mathrm{M}}$ represents the rotation matrix from $F_{M}$ to $F_{O}$, $\left[{ }^{O} x_{M}, O_{y_{M}}\right]^{T}$ is the position of $M$ in $F_{O} \cdot \theta$ is the estimated heading angle of the vehicle.

The position ${ }^{O} X_{i}$ depends on five variables: the $2 \mathrm{D}$ estimated pose $\left({ }^{O} x_{M},{ }^{O} y_{M}, \theta\right)$ of the vehicle in $F_{O}$, the position $\left({ }^{M} x_{i},{ }^{M} y_{i}\right)$ of the cell in $F_{M} \cdot\left({ }^{M} x_{i},{ }^{M} y_{i}\right)$ has no uncertainty because the positions of the cells are known. Thus, the position uncertainty of the cell in $F_{O}$ comes only from the 2D estimated pose of the vehicle $\left({ }^{O} x_{M},{ }^{O} y_{M}, \theta\right)$.

To understand the effect of the uncertainty propagation, let us suppose that the heading angle $\theta$ is decorrelated from the 
position $\left({ }^{O} x_{M},{ }^{O} y_{M}\right)$. This allows analyzing the influence separately.

Firstly, suppose the heading angle has no uncertainty. The relation of the cell position ${ }^{O} X_{i}$ with the position of the vehicle is linear, thus:

$$
\operatorname{Var}\left({ }^{O} X_{i} \mid \operatorname{var}(\theta)=0\right)=\operatorname{Var}\left(\left[o_{x_{M}},{ }^{O} y_{M}\right]^{T}\right),
$$

which means that the covariance of the position of the cell is identical to the covariance of the vehicle position.

Now, suppose the heading angle has some uncertainty and the position is perfectly known. The covariance matrix of ${ }^{O} \mathbf{X}_{i}$ can be computed in closed form as:

$$
\begin{array}{r}
\operatorname{Var}\left({ }^{O} \mathbf{X}_{i} \mid \operatorname{Var}\left(\left[\begin{array}{c}
O_{x_{M}} \\
O_{y_{M}}
\end{array}\right]\right)=0\right)=\left[\frac{d^{\mathbf{O}} \mathbf{X}_{\mathbf{i}}}{d \theta}\right] \cdot \operatorname{var}(\theta) \cdot\left[\frac{d^{\mathbf{O}} \mathbf{X}_{\mathbf{i}}}{d \theta}\right]^{T} \\
=\operatorname{var}(\theta) \cdot\left[\begin{array}{cc}
u(\theta) & t(\theta) \\
t(\theta) & v(\theta)
\end{array}\right]
\end{array}
$$

where

$$
\begin{gathered}
u(\theta)=\left(-\sin (\theta) \cdot{ }^{M} x_{i}-\cos (\theta) \cdot{ }^{M} y_{i}\right)^{2} \\
v(\theta)=\left(\cos (\theta) \cdot{ }^{M} x_{i}-\sin (\theta) \cdot{ }^{M} y_{i}\right)^{2}
\end{gathered}
$$

$t(\theta)=\sin \theta \cdot \cos \theta \cdot\left(\left({ }^{M} y_{i}\right)^{2}-\left({ }^{M} x_{i}\right)^{2}\right)+{ }^{M} x_{i} \cdot{ }^{M} y_{i} \cdot\left((\sin \theta)^{2}-(\cos \theta)^{2}\right)$

The uncertainty in this case is a function of $\left({ }^{M} x_{i},{ }^{M} y_{i}\right)$, which means that the position of the cell in $F_{M}$ determines the shape of the uncertainty in $F_{O}$. The farther a cell is located, the larger the uncertainty is in the $x$ direction. The uncertainty in the $y$ direction (with the same $y$ coordinate) increases when $x$ coordinate augments. Thus, in general, we can conclude that the uncertainty of one cell due to heading error increases in the $x$ direction.

For the general case, let denote $\operatorname{Var}\left({ }^{O} \mathbf{X}_{i}\right)$ the uncertainty of cell $i$ in the global frame. $f\left({ }^{O} x_{M},{ }^{O} y_{M}, \theta\right)$ denotes the transformation (Eq 2). At the first order, we have:

$$
g_{i}(x, y)=\operatorname{Var}\left({ }^{O} \mathbf{X}_{i}\right)=\left[\frac{\delta f}{\delta^{O} \mathbf{X}_{i}}\right] \cdot{ }^{O} \mathbf{P} \cdot\left[\frac{\delta f}{\delta^{O} \mathbf{X}_{i}}\right]^{T},
$$

where ${ }^{O} \mathbf{P}$ represents the covariance matrix of the $2 \mathrm{D}$ pose $\left({ }^{O} x_{M},{ }^{O} y_{M}, \theta\right)$ and $\left[\frac{\delta f}{\delta^{O} \mathbf{X}_{i}}\right]$ the Jacobian.

\section{LANE BELIEF CONSTRUCTION}

The lane belief distribution characterizes the probability of every lane to be the one in which the vehicle is located based on the estimated pose, according to the road rules stored in the map. For this purpose, a multi-hypothesis probabilistic approach is proposed. So, a lane belief serves both for the probabilistic and evidential approaches in the grid cell belief calculation process. In general, the belief level is denoted as $B()$ in this part, which can be transformed to $P()$ and $m()$ in the probabilistic and evidential approaches respectively. Let $B(i, A)$ be the belief of lane $i$ being state $A$.

\section{A. Multi-hypothesis approach}

One can deduce from the pose estimation every possibility concerning which lane is Ego. The method considers every lane where the host vehicle can be located, then computes for each case the belief supporting that particular hypothesis.

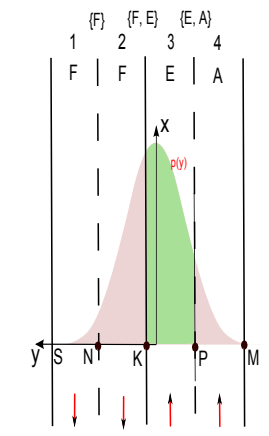

(a) Hypothesis one : 3 is Ego lane

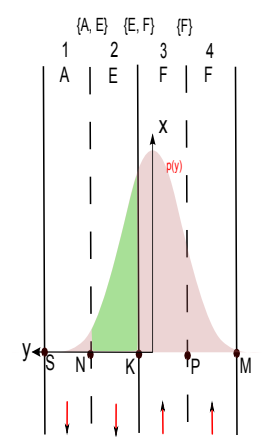

(c) Hypothesis two: 2 is Ego lane

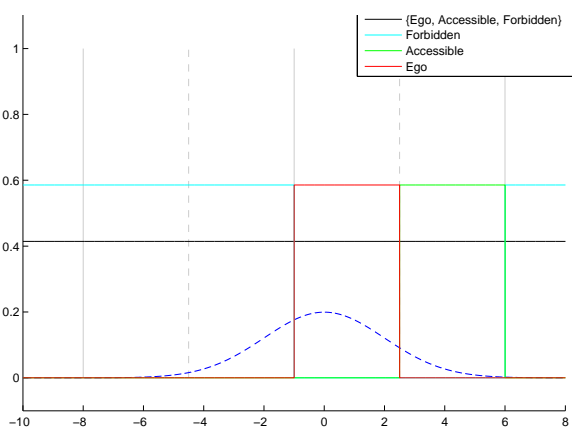

(b) Belief distribution

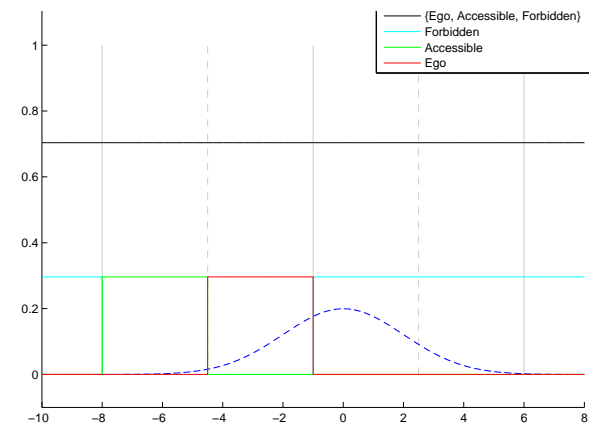

(d) Belief distribution

Figure 4: Multi-hypothesis algorithm illustration

In Fig 3. $p(y)$ represents the lateral position distribution in $F_{R}$. If the position has a large lateral uncertainty, the Gaussian distribution covers several lanes. Fig 4 gives two illustrative examples (corresponding to two hypotheses) on a four-lane road with solid lane marking in the middle ( $A$ refers to Accessible, E Ego and F Forbidden). We herein take the two hypotheses in Fig 4 as examples to illustrate the approach, considering each hypothesis is tackled similarly. A resultant belief distribution considering all hypotheses will be given in section IV-B In Fig 4a, lane 3 is assumed to be $E g o$ and then the belief is calculated as the integral of the pose distribution over the lane. Thus, $B(3, E g o)=$ $P($ Lane $\{3\}=E g o)=\int_{K}^{P} p(y) d y$. This belief is propagated to the other lanes based on the hypothesis that the map is accurate and with no attribute error. Once lane 3 is regarded as $E g o$, lane 4 should be Accessible with the same amount of belief based on the relationship of the two lanes on the map, $B(4$, Accessible $)=P($ Lane $\{3\}=E g o)$. The same rule applies to lane 1 and lane 2 . These two lanes can only be Forbidden, thus $B(1$, Forbidden $)=B(2$, Forbidden $)=$ $P($ Lane $\{3\}=E g o)$. The hypthesis in Fig $4 \mathrm{c}$ is tackled with the same reasoning.

A computation is given in the same road situation. The results are shown besides each possibility in Fig 4 . Here, we do not ignore the possibility that the true pose lies outside 
the road. In contrast, the two regions outside the road (on the left and right) are considered as two independent spaces. This process guarantees the unity of the calculated belief.

\section{B. Belief accumulation}

Based on the illustration above, different hypotheses can have distinct opinions on the states of the same lane space. For instance, the first example in Fig 4 a brings Forbidden belief for lane three, whereas the second example in Fig $4 \mathrm{c}$ computes the Ego belief for this lane. These two types of belief are both stored in the belief distribution of the lane. If other hypotheses would have contributed to the same proposition, the belief would be accumulated. This computation preserves the belief from all possible hypotheses. The final belief distribution is shown in Fig 5 The belief accumulation is mostly highlighted from the Forbidden mass outside the road. The lateral pose distribution is limited to the road area, thus Forbidden belief accumulates to $100 \%$ outside the road, which is conform with reality.

Every hypothesis is tackled independently and brings new belief to the space on different propositions.

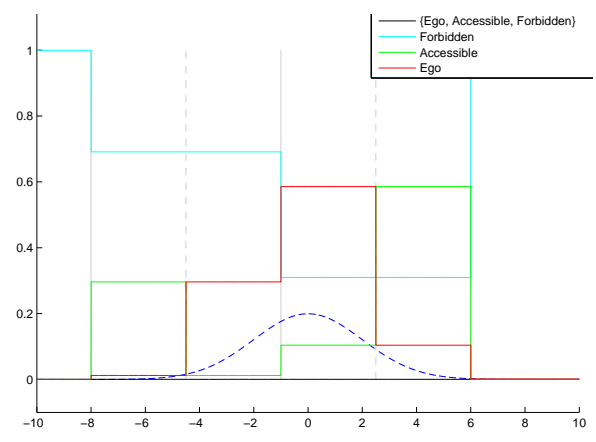

Figure 5: Final lane belief distribution in the cross-track direction

\section{GRID CELL BELIEF CALCULATION}

With the lane probability distribution already estimated, the formalism to propagate the belief state in every cell is detailed, for both probabilistic and evidential approaches.

\section{A. Probabilistic approach}

To take advantage of the lane belief distribution constructed in section IV the probability of lane $i$ to be in the state $A$ is $P\left(S_{l_{k}}=A\right)=B(k, A)$.

Let define two properties for each cell in the grid: $L_{i}$ and $S_{i}$. They indicate respectively the lane index and the state of cell $i$. Thus $L_{i} \in(1,2, \ldots n)$ where $n$ represents the number of lanes, and $S_{i} \in \Omega$

The probability of the red cell $i$ in Fig 1 being located in lane $k$ can be calculated as:

$$
P\left(L_{i}=k\right)=\iint_{\left(x, y \in \text { Lane }_{k}\right)} g_{i}(x, y) d_{x} d_{y} .
$$

According to the total probability law, the probability of the state $A$ for the cell is computed as:

$$
P\left(S_{i}=A\right)=\sum_{k=1}^{n} P\left(S_{i}=A \mid L_{i}=k\right) \cdot P\left(L_{i}=k\right), A \in \Omega .
$$

With $P\left(L_{i}=k\right)$ already tackled in Eq. 5, the problem resides now in computing $P\left(S_{i}=A \mid L_{i}=k\right)$. Suppose the state for lane $k$ is denoted as $S_{l_{k}}$. The first part can be developed into:

$$
P\left(S_{i}=A \mid L_{i}=k\right)=P\left(S_{i}=A \mid S_{i}=S_{l_{k}}\right)
$$

because if one cell lies in lane $k$, then it has the same state as the lane $k$

Moreover,

$$
P\left(S_{i}=A \mid S_{i}=S_{l_{k}}\right)=P\left(S_{l_{k}}=A\right)=B(k, A) .
$$

This process is repeated for every cell in the grid.

\section{B. Evidential approach}

Dempster-shafer's theory provides a generalized way to deal with uncertainty. The singletons in $\Omega$ are mutually exclusive. The power set is defined as:

$$
2^{\Omega}=\{\emptyset, \text { Ego, Accessible, Forbidden, }\{\text { Ego, Accessible }\},
$$

$\{$ Ego, Forbidden $\},\{$ Accessible, Forbidden $\}, \Omega\}$.

One advantage of the evidential representation is that one can attribute mass to any subset of the frame of discernment. For example $\{E g o$, Accessible $\}$ is not null in situations where the mass supports both Ego and Accessible, but there is not enough information to tell whether the mass should be assigned to Ego or Accessible.

If one cell lies inside one lane, then it should have the same mass distribution as the lane.

$$
m^{i}=m_{k}, \text { if } C_{i} \in \text { Lane }_{k}
$$

$m^{i}$ and $m_{k}$ represent respectively the mass distribution of cell $i$ and Lane $_{k}$. Note that the belief distribution constructed in section IV can be adopted here as $m_{k}(A)=B(k, A)$.

Due to the position uncertainty $g_{i}(x, y)$, the cell $i$ can be located within each lane with a certain confidence level computed by:

$$
\alpha_{k}^{i}=\iint_{\left(x, y \in \text { Lane }_{k}\right)} g_{i}(x, y) d_{x} d y .
$$

This confidence level is seen as a degree of reliability of the lane state decision and can be used to discount the mass distribution of each lane $m_{k}$ [12]. The mass distribution for the cell $i$ belonging to lane $k$ is:

$$
\begin{gathered}
m_{k}^{i}(A)=\left(1-\alpha_{k}^{i}\right) m_{k}(A), A \neq \Omega \\
m_{k}^{i}(\Omega)=\left(1-\alpha_{k}^{i}\right) m_{k}(\Omega)+\alpha_{k}^{i}
\end{gathered}
$$

Fusing the information provided by all lanes, we can now compute the mass distribution for cell $i$ by:

$$
m^{i}=\odot_{k} m_{k}^{i}, k=1,2, \ldots, n
$$

where $k$ is the lane index, $n$ is the number of lanes.

The fusion operator $\odot$ proposed in [13] is defined as:

$\left\{\begin{array}{c}\left(m_{1} \odot m_{2}\right)(A)=\sum_{B \cap C=A \neq \emptyset} m_{1}(B) \cdot m_{2}(C) \\ \left(m_{1} \odot m_{2}\right)(A)=\sum_{B \cap C=\emptyset, B \cup C=A} m_{1}(B) \cdot m_{2}(C)\end{array} \quad A, B, C \subset \Omega\right.$ 
The specialty of this operator is that the conflicting mass is put into union states. In our case, this conflicting mass is due to the position uncertainty. If, in the fusion process, conflicting information is generated, we assume that the evidence supports the mass distribution of the two fused hypotheses. This highlights an advantage of the evidential approach which will be demonstrated in the results.

\section{REAL ROAD EXPERIMENTS AND RESULTS}

Real road experiments have been carried out with an equipped vehicle of the Lab shown in Fig 6 A map with good accuracy has been used. In the map, any road is explicitly described with lane information, including lane markings and road boundaries. The lane markings are distinguished in the map with different attributes. This feature provides semantic information. A GPS system with RTK corrections provided accurate positioning information with high confidence during the tests.

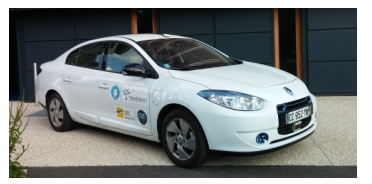

Figure 6: Robotex experimental vehicle

To qualitatively evaluate the result, the approach proposed in [14] is adopted. A wide-angle scene camera was installed behind the windshield during the acquisition process. The retro-projection of the Lane Grid on the scene image helps supporting a qualitative evaluation. The essential purpose of this method is to analyze and evaluate the correspondence between the Lane Grid and the scene observed by the camera.

The grids are of $40 * 16$ meters in length and width and the cells size is $(0.1 * 0.1)$ meters. The whole approach has been implemented in $\mathrm{C}++$. In the following, we focus on one particular sample and we compare the two approaches. The results are shown in the form of a RGB image which represents the lane grid retro-projected on a front-looking camera. The advantage is that the belief level is reflected by the RGB color brightness. A brighter color means a higher believe level.

\section{A. Probabilistic lane grid result}

Fig 7 shows the resulting probabilistic grids with the following pose uncertainty $\left(\sigma_{x}=0.3 m, \sigma_{y}=0.2 m, \sigma_{\theta}=\right.$ 0.1 radians). The Ego, Accessible, and Forbidden probabilities along each lane become smaller as the distance of the cells to the host vehicle gets larger. However, the space covered by the probability distribution extends outside of each lane, which means that the probability becomes more dispersed. The uncertainty propagation explained in section III is the reason for this phenomenon. It is clear that if one cell has a larger position uncertainty, it ought to have more ambiguous probability distribution. As the

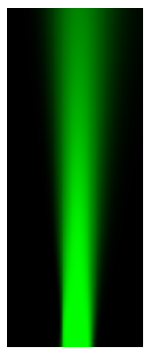

(a) Ego

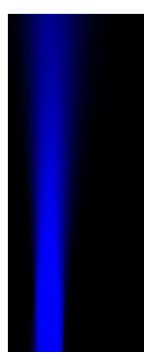

(b) Accessible

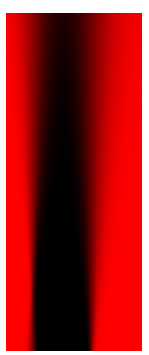

(c) Forbidden

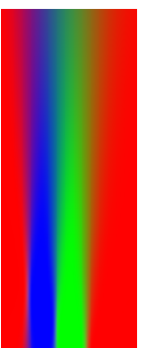

(d) All

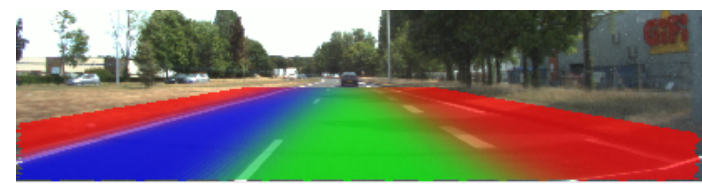

(e) Retro-projection on the front-looking image

Figure 7: Probabilistic approach

approach takes into account the $2 \mathrm{D}$ pose uncertainty, the cells at farther distance tend to have larger uncertainty. The combined probability shown in Fig $7 \mathrm{~d}$ further reflects this phenomenon. One can remark clearly that cells close to the host vehicle tend to have just single state probability, whereas at farther distance, the probability distribution can become very ambiguous.

The retro-projection of the lane grid on the image in Fig $7 \mathrm{e}$ gives a qualitative result evaluation. One can see the result is valid given the correspondence of the lane grid projected on the image.

\section{B. Evidential lane grid result}

Fig 8 displays the results of the evidential approach with the same pose uncertainty. The belief of the states of the lanes gradually decreases as the distance of the cells to the host vehicle becomes larger. From the mass in the union states shown in Fig 8d, one can remark that some quantity of mass is put in the union states, which is displayed by the combination of colors. In Fig 8d, yellow, cyan and magenta colors represent respectively the mass in $\{E g o$, Forbidden $\}$, $\{$ Ego, Accessible $\}$ and $\{$ Accessible, Forbidden $\}$. The fact that farther cells have larger uncertainty is more clearly reflected in this image, as the union mass area becomes wider in the farther space. In Fig $8 \mathrm{~g}$, the retro-projection of union mass is displayed. This result directly shows the fact that the union mass is mainly focused on the cells that are close to the lane markings.

\section{Influences of position and angle uncertainties}

The position uncertainty is propagated uniformly over the lane grid, whereas the angle uncertainty is not. We herein show the results reflecting these properties. This effect have the same impact on the Bayesian and Evidential approaches, herein the evidential results are shown for illustration since 


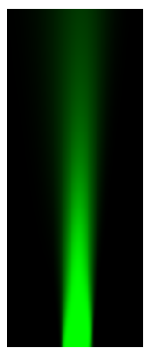

(a) Ego

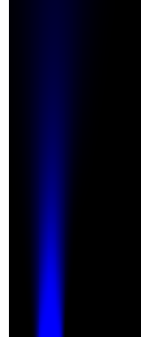

(b) Accessible

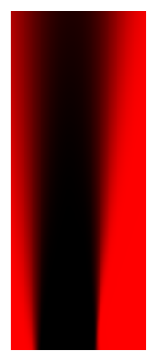

(c) Forbidden

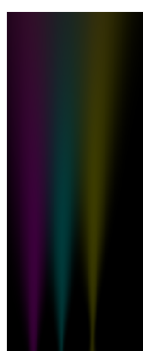

(d) Union

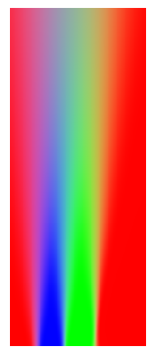

(e) Singleton

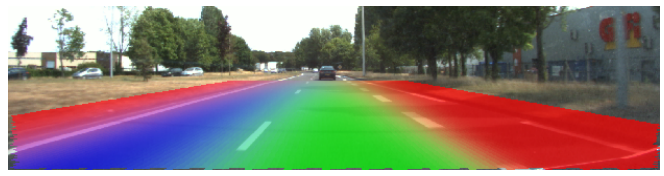

(f) Retro-projection of all singleton masses on the image

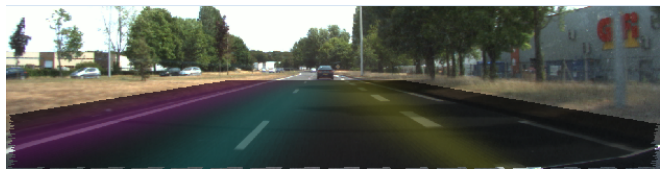

(g) Retro-projection of the union of masses on the image

Figure 8: Evidential approach

more visual results can be demonstrated. In Fig 9 the results with only position uncertainties are displayed. The uncertainties are respectively $(0.3 \mathrm{~m}, 0.2 \mathrm{~m}, 0.0$ radians $)$ and $(1.0 \mathrm{~m}, 1.0 \mathrm{~m}, 0.0$ radians $)$ in Fig $9 \mathrm{a}$ and Fig $9 \mathrm{~b}$ WIth position uncertainty, the fading effect of each lane states over each lane is uniform all along the lane space. With larger position uncertainty, the mass level for lane states get lower, which means less confident about the lane states.

The Fig 10 shows two cases with different angle uncertainties, and with no position uncertainty. The uncertainties are respectively $(0.0 \mathrm{~m}, 0.0 \mathrm{~m}, 0.05$ radians $)$ and $(0.0 \mathrm{~m}, 0.0 \mathrm{~m}, 0.1$ radians $)$ in Fig $10 \mathrm{a}$ and Fig $10 \mathrm{~b}$. The effect of larger angle uncertainty is reflected by the lower mass level over the space in larger distance in Fig $10 \mathrm{~b}$ With larger angle uncertainty, the cells in Fig $10 \mathrm{~b}$ have larger uncertainties, thus their mass dispersion is more significant, which results in the more ambiguous lane grid.

\section{Comparison between the two approaches}

The evidential approach provides richer information than the probabilistic one. Indeed, it puts beliefs into union states if the belief in each single state is not clear. Ignorance is explicitly quantified by the Unknown mass. Moreover, the evidential approach provides a flexible method to tackle conflicting information which also brings useful information. Here, the conflicting mass corresponds to the lane markings.

Another essential difference resides in the decision process. With a probabilistic approach, decision is classically made by selecting the maximum probability. Thus, the decision is always among these three states, no matter how
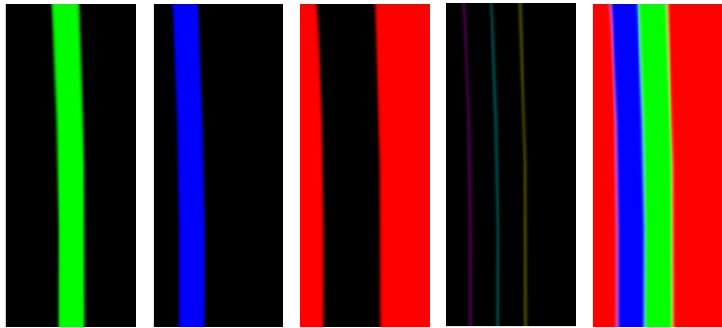

(a) small position uncertainty
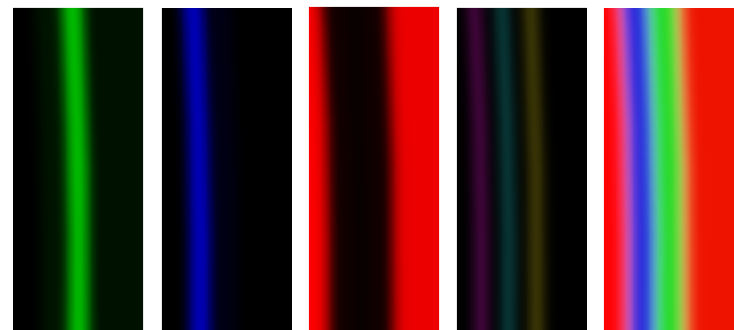

(b) large position uncertainty

Figure 9: Results with only position uncertainties
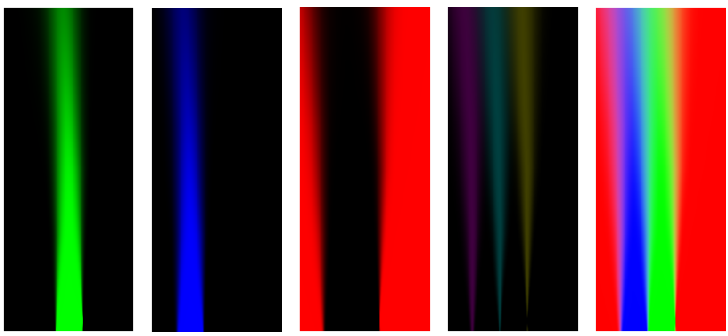

(a) small angle uncertainty
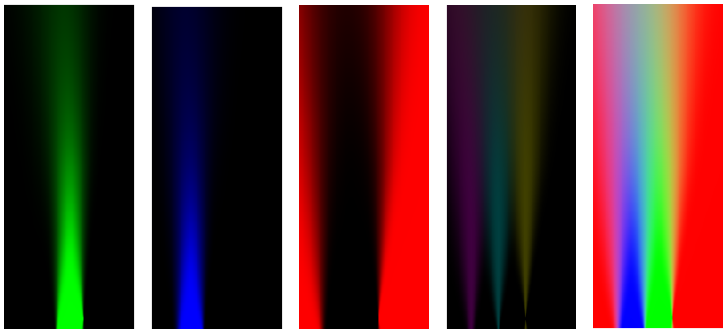

(b) large angle uncertainty

Figure 10: Results with only angle uncertainties

uncertain the information is. However, with the evidential approach, it is authorized to explicitly announce ignorance when there is too large uncertainty. Fig 11 shows the decision grids of the results shown in Fig 7 and in Fig 8 . In these decision grids, the belief level is kept to reflect the uncertainty level. Fig 11a shows the max of probabilities. The Ego and Accessible lanes at farther distance become more narrow. This phenomenon is due to the fact that the probability dispersion in this area becomes larger and the Forbidden belief outside the road has larger influence which leads to Forbidden belief invasion into lane space. Fig $11 \mathrm{~b}$ shows a decision grid based on the maximum of mass of evidence. 


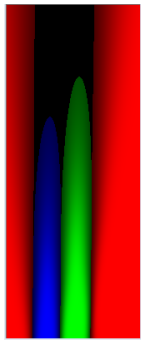

(a) Probability (b) Evidence

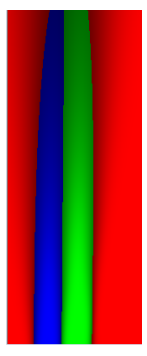

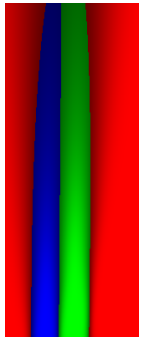

(c) Pignistic
Figure 11: Decision grids (used by client applications)

One can remark that over the far away space, the cells are Unknown, which means that no decision can be made in this area due to the lack of information. This is a great advantage for a path planning process, since, as this evidential decision grid provides explicitly ignorant information, no risky trajectory can be planned.

In Fig 11c shows another decision grid based on pignistic probability [15], commonly used to transform evidence masses in probabilities. One can remark that this grid looks almost exactly the same as the probabilistic decision grid in Fig 11a In fact, the ratio of identical decision of these two grids reaches $99.992 \%$. This result validates the way uncertainty is handled in the proposed evidential grid mechanism as it conducts to the same probabilistic conclusions.

\section{E. Average construction time study}

Herein a study of time consumption of the evidential lane grids based on the cell resolution variation is presented. Above results are shown with cell resolution at 0.1 meters, which is really for the purpose of showing the grid properties. In Figure 12 the time consumption variation with regards to the lane grid resolution is illustrated by a simple plot. The size of the grids keeps at ( $40 \times 16$ meters). This average time is computed along a trajectory of 26 seconds including 260 frames of data using a common personal computer. According to this figure, One can remark that the average time consumption decreases exponentially with regards to the cell size augmentation.

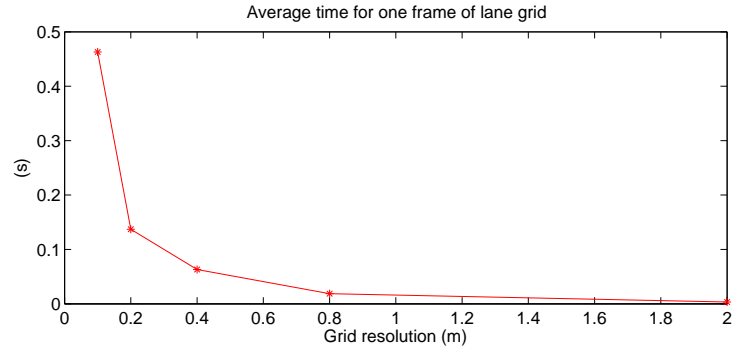

Figure 12: Average time for evidential lane grid construction with different resolutions

\section{CONCLUSION}

A new approach to characterize lane information and semantic road rules into grid cells has been proposed. A prior lane-level map is used to extract lane information based on the pose provided by a localization system. As any localization system provides uncertain information, the uncertainty propagation over the grid has been analyzed and methods to build probabilistic and evidential lane grids have been proposed. Real road results have been reported and compared. The evidential framework relying on mass discounting has been validated through decision grids comparisons. It has the advantage of explicitly managing ignorance which is clearly an added value for safe autonomous navigation.

\section{Video}

A video illustrating the results of a whole test on public roads with lane changes is accessible on line 1 It provides a qualitative evaluation of the method.

\section{REFERENCES}

[1] J. M. Alvarez, T. Gevers, Y. LeCun, and A. M. Lopez, "Road scene segmentation from a single image," ECCV'12 Proceedings of the 12th European conference on Computer Vision, vol. 14, pp. 376-389, 2012.

[2] S. Wang, S. Fidler, and R. Urtasun, "Holistic $3 d$ scene understanding from a single geo-tagged image," International Conference on Computer Vision and Pattern Recognition (CVPR), Boston, pp. 3964 3972, 2015.

[3] J. M. Alvarez, A. M. Lopez, T. Gevers, and F. Lumbreras, "Combining priors, appearance, and context for road detection," IEEE Transactions on Intelligent Transportation Systems, vol. 15, pp. 1168 - 1178, 2014.

[4] M. Aly, "Real time detection of lane markers in urban streets," IEEE Intelligent Vehicles Symposium, vol. 6, pp. 7 - 12, 2008.

[5] A. Parajuli, M. Celenk, and H. B. Riley, "Robust lane detection in shadows and low illumination conditions using local gradient freatures," Open Journal of Applied Sciencecs, vol. 7, pp. 68 - 74, 2013.

[6] G. Cui, J. Wang, and J. Li, "Robust multilane detection and tracking in urban scenarios based on lidar and mono-vision," IET Image Processing, vol. 8, pp. $269-279,2013$.

[7] A. Schindler, "Vehicle self-localization with high-precision digital maps," IEEE Intelligent Vehicles Symposium (IV), pp. 141 - 146, 2013.

[8] Z. Tao and P. Bonnifait, "Road invariant extended kalman filter for an enhanced estimation of gps errors using lane markings," IEEE/RSJ International Conference on Intelligent Robots and Systems, pp. 3119 - 3124, 2015.

[9] A. Elfes, "Using occupancy grids for mobile robot perception and navigation," Computer, vol. 22, no. 6, pp. 46 - 57, 1989.

[10] D. Pagac, E. M.Nebot, and H. Durrant-Whyte, "An evidential approach to map-building for autonomous vehicles," IEEE Transactions on Robotics, vol. 7, pp. 623-629, 1998.

[11] J. Moras, V. Cherfaoui, and P. Bonnifait, "Credibilist occupancy grids for vehicle perception in dynamic environments," IEEE International Conference on Robotics and Automation, pp. 84 - 89, 2011.

[12] G. Shafer, A Mathematical Theory of Evidence, P. U. Press, Ed. Princeton University Press, 1976.

[13] D. Dubois and H. Prade, "Representation and combination of uncertainty with belief functions and possibility measures," Computer intelligence, vol. 4, pp. 244-264, 1988.

[14] J. Moras, S. A. R. F., V. Drevelle, G. Dherbomez, V. Cherfaoui, and P. Bonnifait, "Drivable space characterization using automotive lidar and georeferenced map information," Intelligent Vehicles Symposium, pp. $778-783,2012$.

[15] P. Smets, "Decision making in the tbm: the necessity of the pignistic transformation," Int. Journal of Approximate Reasoning, vol. 38, pp. 133-147, 2005.

${ }^{1}$ https://youtu.be/hftEkFtrKw8 